Article

\title{
Barriers to the Adoption of Urban Living Labs for NBS Implementation: A Systemic Perspective
}

\author{
Shahryar Sarabi ${ }^{1, *}\left(\mathbb{D}\right.$, Qi Han $^{1}$, A. Georges L. Romme ${ }^{2}{ }^{\circledR}$, Bauke de Vries ${ }^{1} \oplus$, Rianne Valkenburg ${ }^{2}$, \\ Elke den Ouden ${ }^{2}$, Spela Zalokar ${ }^{3}$ and Laura Wendling ${ }^{4}$ \\ 1 Information Systems in the Built Environment (ISBE) Group, Department of Built Environment, \\ Eindhoven University of Technology, Groene Loper 3, 5612 AE Eindhoven, The Netherlands; \\ q.han@tue.nl (Q.H.); b.d.vries@tue.nl (B.d.V.) \\ 2 Department of Industrial Engineering \& Innovation Sciences, Eindhoven University of Technology, \\ Groene Loper 3, 5612 AE Eindhoven, The Netherlands; a.g.l.romme@tue.nl (A.G.L.R.); \\ a.c.valkenburg@tue.nl (R.V.); e.d.ouden@tue.nl (E.d.O.) \\ 3 ENoLL, Pleinlaan 9, 1050 Ixelles, Belgium; spela.zalokar@enoll.org \\ 4 VTT Technical Research Centre Ltd., 02150 Espoo, Finland; laura.wendling@vtt.fi \\ * Correspondence: s.ershad.sarabi@tue.nl
}

check for updates

Citation: Sarabi, S.; Han, Q.; L. Romme, A.G.; de Vries, B.;

Valkenburg, R.; den Ouden, E.;

Zalokar, S.; Wendling, L. Barriers to the Adoption of Urban Living Labs for NBS Implementation: A Systemic Perspective. Sustainability 2021, 13, 13276. https://doi.org/10.3390/ su132313276

Academic Editor: Baojie He

Received: 18 October 2021

Accepted: 26 November 2021

Published: 30 November 2021

Publisher's Note: MDPI stays neutral with regard to jurisdictional claims in published maps and institutional affiliations.

Copyright: (c) 2021 by the authors. Licensee MDPI, Basel, Switzerland. This article is an open access article distributed under the terms and conditions of the Creative Commons Attribution (CC BY) license (https:/ / creativecommons.org/licenses/by/ $4.0 /)$.

\begin{abstract}
Urban Living Labs (ULLs) are widely believed to provide a safe environment for experimentation, co-creation and evaluation of innovations in real-life settings. A growing number of cities have been adopting ULLs to co-create and test Nature-Based Solutions (NBS). However, many of these cities have been facing major barriers in trying to adopt the ULL approach for implementing NBS. In this study, we seek to identify these barriers and provide a systemic understanding. Barriers are identified by means of workshops and interviews. Subsequently, interpretive structural modelling serves to identify the interdependencies among the barriers, resulting in a structural model of barriers in adopting ULLs for NBS. Our results show that political and institutional barriers are significantly limiting the adoption of ULLs. Moreover, knowledge brokers and other intermediaries, as well as cross-sectoral collaboration, play a key role in getting ULLs adopted. The findings from this study can help cities to develop strategies that overcome the main barriers for ULL adoption in the context of nature-based solutions.
\end{abstract}

Keywords: urban living lab; nature-based solutions; NBS; barriers; interpretive structural modelling; urban experiments

\section{Introduction}

Nature-based solutions (NBS) are being increasingly adopted by cities in response to climate change as well as to improve urban resilience [1,2]. NBS are inspired by, copied from, and/or supported by nature [3] and are implemented to solve or mitigate multiple societal problems [4-6]. As such, NBS are inter- and transdisciplinary innovations that deal with different social, ecological and technological aspects of urban environments. Cities therefore need to adopt novel approaches for facilitating the interaction between different stakeholders and supporting the development of innovative solutions. Here, Urban Living Labs (ULLs) are being increasingly adopted as a promising approach for developing NBS, especially in many cities across Europe $[7,8]$. ULLs appear to provide a flexible governance approach that enables experimentation, learning, reflexivity and reversibility in developing and implementing NBS [9]. In other words, ULLs are often introduced to orchestrate the collaboration between multiple stakeholders-including companies, research communities, public sector representatives and citizens-by providing a suitable context for co-creation [10].

Despite these benefits of adopting ULLs, there is also initial evidence about the multiple barriers that various cities have faced while adopting the ULL approach [11,12]. 
However, these barriers have not yet been systematically mapped, despite the need for a deeper understanding in this area. In this paper, we aim to identify, explore and analyse the barriers to the adoption of ULLs for NBS implementation by studying three front-runner cities participating in the UNaLab project (a European Horizon 2020 project): Tampere, Eindhoven and Genoa. These three European cities have been developing ULLs for NBS within this collaborative project, and their collective experience can be insightful for other cities exploring this path.

Notably, barriers to the adoption of urban innovations can be highly interdependent, and recognizing these relationships may be critical for developing effective urban strategies [13]. We therefore employ Interpretive Structural Modelling (ISM) and MICMAC analysis to provide a systemic understanding of the barriers.

This paper is structured as follows. In Section 2, the three city contexts are introduced. Section 3 then serves to identify the common barriers in these cities. In Section 4, the ISM approach is described and applied to the barriers previously identified. Our main findings are discussed in Section 5, and a conclusion is provided in the last section.

\section{UNaLab Urban Living Labs}

Three front-runner cities in the UNaLab project have been developing NBS using the ULL approach. In this section, the local context of each of these cities is shortly presented.

\subsection{Tampere}

The city of Tampere in Finland primarily aims to develop NBS to address stormwater management issues. Vuores and Hiedanranta are the two main living labs for NBS in Tampere. Vuores is a neighbourhood located in a natural area that was zoned for housing in 2007. Ambitious sustainability goals were set for this neighbourhood to compensate for the human impact on this natural area. Hiedanranta is an old pulp mill area without any population currently residing there. The municipality of Tampere plans to construct housing for 25,000 people in this neighbourhood in the next 30 years.

The following NBS were implemented in the context of the UNaLab project in Vuores: retention basins, alluvial meadows, urban gardens with small-scale NBS and wooden boardwalks (duckboards) to improve the citizens' access to natural areas. In Hiedanranta, a green wall, biofilters and microalgae-based wastewater treatment system were selected as the NBS measures. In both the Vuores and Hiedanranta ULLs, the co-creation process started by developing a shared understanding of NBS and their benefits. This co-creative approach gave rise to valuable lessons for all stakeholders. For example, the complications occurring after the implementation of biofilters in Hiedanranta enhanced the stakeholders' awareness and knowledge regarding the technical, institutional and social aspects of adopting NBS [14]. More generally speaking, this type of mutual learning appeared to be one of the main benefits of the co-creation process in Tampere [14].

\subsection{Eindhoven}

The city of Eindhoven (Netherlands) planned to implement several NBS including a green roof on the City Hall and the re-vegetation of Clausplein using the ULL approach. These two ULLs and their NBS actions have been fully implemented. The staff of the municipality was already familiar with both the notion of NBS and co-creation processes before starting these NBS implementations. Likewise, many citizens were also experienced in co-creation processes. However, the co-creation sessions in the context of the UNaLab project mainly involved relevant professionals (from the municipality and industry) in order to generate a local community of practice around NBS.

Co-creation has long been a critical factor in the city of Eindhoven, not only because it is mandated by Dutch law, but also because it suits the local culture and is demanded by many stakeholders $[15,16]$. The NBS co-creation activities were organized with the aim to develop a practical plan which would serve to implement NBS in a relatively short period. In these co-creation processes, various challenges were identified, suitable solutions were 
selected, and a shared understanding of how different municipal departments should collaborate was established.

\subsection{Genoa}

Genoa has approved a refurbishment plan for the former Gavoglio Barracks located in the Lagaccio district. The city is introducing a range of different NBS in this area, mainly to enhance the resilience to flooding and urban heat risk. The NBS and supporting measures include infiltration basins, rain gardens, trees, vegetated gabions, permeable pavements and others.

According to previous surveys conducted in Genoa, the familiarity with NBS and cocreation practices was lower in comparison to Tampere and Eindhoven [16]. The regulations that support and encourage stakeholder consultation in Genoa are largely limited to informing citizens about planned and on-going projects. Thus, the city was less experienced in active co-creation with citizens and involving various other stakeholders in planning and decision-making. The ULL co-creation process in Genoa therefore aimed to develop a shared vision, elaborate project ideas about creating an urban park and share knowledge about NBS among the stakeholders.

\section{Barriers to the Adoption of Urban Living Labs for NBS Projects \\ 3.1. Barrier Identification Workshops and Interviews}

All three cities have experienced various barriers in adopting ULLs for developing and implementing NBS. This section presents the barriers found throughout a series of interviews and workshops with experts and stakeholders involved in the co-creation processes in these cities. Two types of workshops were arranged to identify and analyse the barriers: a series of city-specific workshops and interviews and one collective workshop involving experts from all cities participating in the UNaLab project.

The workshop in Eindhoven was conducted on 29 October 2019 including 11 experts from different organisations and institutional levels. The actors involved were from various municipal departments, the local water board, the province of Noord-Brabant and several citizens with sustainability interests. The workshop in Tampere was conducted on 9 June 2020 with six experts from the municipality. This workshop was in an online format in view of the COVID-19 situation in Europe at the time. Due to the severe restrictions caused by the pandemic situation, we conducted one-to-one online interviews with three experts coordinating the co-creation activities in Genoa.

The collective workshop was conducted on 28 November 2019 in Castellón de la Plana, Spain. This workshop involved 28 experts from different cities and institutions. In this workshop, the most common barriers were identified, and the relationships among these barriers were analysed.

\subsection{Identified Barriers}

Several barriers were identified during the workshops and interviews, and an initial list of barriers was generated. To validate the barriers identified and compare them with previous studies, a literature review was conducted. The literature about ULLs is still relatively new, and few studies in this area have addressed challenges specific to ULL adoption. Therefore, we also studied the literature discussing the barriers to the adoption of related concepts and approaches such as Living Labs, open innovation practices and social innovation arrangements. A total of 45 papers and reports were selected and reviewed.

Different types of barriers were identified during this process, ranging from institutional barriers that municipalities deal with on a daily basis to social and cultural issues arising from the ways that different groups of people think and behave. In line with other studies [17-19], the barriers were categorized into four groups: organisational and structural barriers; cognitive and behavioural barriers; knowledge and process barriers; and ethical barriers. The following subsections outline the resulting barriers and how they affect ULL adoption for NBS. 


\subsubsection{Organisational and Structural Barriers}

- Lack of Political Will and Long-Term Commitment

The ULL collaborative approach with a wide range of different stakeholders engaging in the early stages of the planning process often is not well-supported by politicians and decision-makers. This matter of "political acceptance" becomes even more prominent when NBS is the innovation being implemented, as both NBS and ULL are novel solutions and approaches with associated uncertainties. Politicians usually prioritise issues that are highly visible, such as the job market and housing, and tend to focus on actions that have short-term, rather certain outcomes [20]. Adopting, developing and realising the multiple benefits provided by NBS ULLs is a medium- to long-term process that is usually not well aligned with short-term political cycles. The municipalities of Eindhoven, Tampere and Genoa each developed long-term goals for the transition towards sustainability, aiming at active involvement of various local stakeholders.

- Lack of supportive legal and policy frameworks

An important barrier spotted in the three cities is the inability of legal frameworks to provide incentives for various stakeholders to remain committed to co-creation activities in the long term. Other studies have also observed this barrier [21]. A stable and supportive legal structure is required to provide a safe context for urban stakeholders to share their innovative ideas. The experimental status of ULLs should therefore be safeguarded in legal frameworks that would provide the flexibility required for experimenting with and learning about NBS.

- Disconnection from the mainstream development process

The analysis of cases in Eindhoven, Tampere and Genoa together with a review of the literature also suggests that ULLs are generally not believed to be a key step in the mainstream processes of policy development $[9,11]$. Rather, ULL projects are mainly driven by the availability of external financial sources, with the resulting ULL then being bound to the period and scope of the individual project's budget. ULLs thus have, to date, not been adopted as an integral element of the municipal policymaking and planning processes within the three cities; this clearly limits the ability of (different groups within) the city to adopt them.

\section{- $\quad$ Sectoral silos}

As witnessed in Eindhoven, Tampere and Genoa, each department and institution tends to have its own agenda, policy framework, strategy and responsibility. In this respect, many workshop participants and interviewees emphasised the phenomenon of "sectoral silos" as a significant barrier to effectively communicating between stakeholders and bringing them together at a single table. Adopting ULLs requires different municipal departments and other stakeholders to be actively engaged in the co-creation process. This is even more critical in the case of NBS. As a transdisciplinary solution concept, NBS requires the active participation of representatives from various groups and multiple disciplines in the ULL activities [22]. However, the data from the three cities suggest that especially the collaborative ties between municipal departments are often not sufficiently developed to enable successful collaboration in ULLs for NBS.

- Inflexible hierarchical organisational structure

Another organisational/structural barrier found in the three cities is the lack of managerial discretion in the municipal departments to delineate and adjust their actions and strategies. The established governance approach in civil service organisations of municipalities is characterised by a hierarchical structure. As a result, relatively slow and inflexible bureaucratic processes can limit the adoption of ULLs [11]. ULLs involve citizens, companies, research institutions and universities which can be discouraged from actively engaging as a result of the rigidity of municipal procedures. It therefore often takes a rather 
long time to make decisions and modify plans, which does not resonate well with the experimental agenda of ULLs.

\section{- $\quad$ Lack of sufficient human resources}

Municipalities tend to allocate limited staff resources to co-creation activities [23]. Examples of this barrier were witnessed in the co-creation workshops conducted in Tampere and Eindhoven. Many experts employed in municipal departments complained about the limited amount of time they could commit to their ULLs. The main reason for this problem was that municipal employees were not allowed to spend a lot of time on activities which are not considered as their direct responsibility within the department and are mandated to do.

- Lack of sustainable financial resources

The lack of financial resources for ULLs and similar long-term experimental activities is another common barrier observed in the three cities [11]. For example, UNaLab's ULLs are mostly financed by means of external resources, which are only available as long as the UNaLab project runs. In general, many cities have been excessively focussing on capital investments; they thus lack the business models and financial mechanisms for supporting co-creation activities and underestimate the budget needed for managing and maintaining new infrastructure after the capital investment phase [24].

\subsubsection{Cognitive, Cultural and Behavioural Barriers}

\section{- $\quad$ Negative past experiences}

The negative experiences that stakeholders, particularly municipal authorities, have had with co-creation processes are identified as a cognitive barrier. This barrier was especially evident in the city of Genoa. This city previously had unsuccessful experiences with several participatory approaches, such as participatory budgeting [20]. Such negative experiences can give rise to a substantial fear of failure, which limits the adoption of ULLs despite municipal staff being highly motivated to get citizens involved. These past experiences can significantly contribute to a problematic perception of the co-creation approach in ULLs.

- Perceived complexity of the ULL approach

Another cognitive barrier involves the perception of those responsible for administering municipal processes regarding living lab and co-creation activities. In some instances, experts conceive of NBS as being rather complex and generating several uncertainties and thus perceive ULLs to be an extra complication in developing NBS. As discussed in the collective workshop with representatives from all three cities, some administrators and experts are averse to including people with diverse expectations and thereby could be drawn into a potentially time-consuming or contentious process, whereas others have a negative attitude towards the use of external knowledge (i.e., the not-invented-here syndrome) [25].

- Risk aversion and reluctance to change

Reluctance to change and risk aversion [26] also constitute a relevant barrier. Aversion to risk and change was observed among several municipal employees, especially those dealing with more technical issues. The latter experts tend to have a significant amount of (informal) power in the civil service organisations of municipalities. These experts usually have a strong preference for actions and solutions with clear and predictable outcomes-which tends to be problematic in ULLs.

\section{- Conflicting expectations}

Diverging expectations among stakeholders involved in ULL activities can lead to conflict [27]. Several of such conflicts have been observed in all three case studies. Diverging expectations can arise between different municipal departments, but also between different civil stakeholders. In some cases, citizens participating in co-creation sessions expected swift decisions on concrete projects, which is not compatible with the 
nature of the NBS co-creation process. Diverging expectations among different (groups of) citizens can also create major challenges in ULL processes. For example, in one particular case, prolonged discussions among citizens expressing conflicting opinions about how to design a playground in a local park created a substantial delay.

- Lack of public awareness and engagement

Lack of awareness among citizens is commonly reported as one of the barriers in developing NBS $[28,29]$. During the interviews and workshops, it was mentioned that NBS are often assumed to be (part of) public infrastructure, and many citizens therefore consider the municipality to be solely responsible for developing them. The importance of civic engagement in ULLs for NBS is not always sufficiently clear. The pivotal role of civic engagement was frequently emphasised during the interviews and workshops, particularly with regard to how it enhances the willingness and commitment of local politicians.

- Lack of engagement to take responsibility

Only a few experts from each municipality were responsible for the adoption and implementation of the ULLs. Our data suggest that municipal staff members were often not motivated and committed to take on any leadership roles in the ULLs created. This lack of engagement to take responsibility appears to limit the adoption of ULLs as an innovative approach [30]. Successfully adopting a ULL requires different municipal departments and groups to collaborate in order to work towards a common goal and coordinate different aspects of the process. However, our data point at the somewhat limited ability and willingness of people to play a leading role in ULL development processes. Similarly, the motivation from stakeholders from the private sector to jointly take responsibility with municipal staff was largely lacking in the three cities.

\subsubsection{Knowledge and Process Barriers}

- Uncertainties regarding the added value and benefits of ULLs

The added value that ULLs provide for NBS projects was not clear to many municipal representatives and citizens involved. Especially in case of a small-scale NBS, these participants do not engage on a daily basis with the solution and thus, the benefits of exploiting local knowledge were often not recognised. However, the value of using the local knowledge of stakeholders in ULLs became more evident to many stakeholders in the three cities along the UNaLab project as a result of the deliberate attempts made to uncover and define this value.

- Lack of available guidelines and tools for engagement

In all three cities, there was an explicit demand for guidelines and tools that can help cities to better engage with various local stakeholders. One of the main problems in each city was the inability to find committed (representatives of various) stakeholders. In the ULL co-creation activities, it was not clear for the organisers who had enough motivation to engage and what their motivations might be. The participants were mainly people who had been involved in earlier local planning processes or, as one interviewee called them: "the usual suspects." Finding other stakeholders with different ideas and priorities, who could actively engage in the co-creation process, was challenging. A set of guidelines and tools that can facilitate the identification of the potential benefits provided by various NBS, along with the possible beneficiaries, appears to be required for increasing the engagement of relevant stakeholders.

- $\quad$ NBS monitoring and assessment challenges

Some perceived characteristics of NBS, as the type of innovation developed in ULLs, also constitute a barrier to ULL adoption. For one, the assessment of the performance or impact of NBS is often perceived to be rather difficult [13]. In this respect, there are differences between NBS and other innovations that cities have been exploring and implementing (possibly in other ULLs). Some benefits of NBS (e.g., social cohesion) are not easily 
measurable or may take a long time to become visible, which makes it rather challenging to get citizens and other stakeholders on board [31]. There are two reports published recently which are dedicated to monitoring and evaluation of NBS: NBS performance impact monitoring protocols [32] and an NBS implementation handbook [33]. In addition, recently a comprehensive handbook to guide monitoring and evaluation planning was released by the NBS Impact Evaluation Framework Task Force [5,34]. These outputs reflect significant growth in the body of knowledge on NBS monitoring and evaluation. However, our data imply that more work needs to be done to get local practitioners to adopt this knowledge and associated practices.

- $\quad$ Lack of skilled knowledge brokers

ULLs for NBS are multifunctional and, as such, their planning and implementation is inherently interdisciplinary [35]. Knowledge brokers and intermediaries provide a supportive institutional space, facilitate networking between different departments and connect stakeholders across scales; knowledge brokers and intermediaries are therefore essential for adopting ULLs and developing and monitoring NBS [5]. They have especially been observed to facilitate mutual learning and conflict management among stakeholders [36].

- Inability to upscale and replicate projects

The outcomes obtained from NBS living labs tend to be highly context-specific, and replicating any learnings can therefore be very challenging [11]. Although it is too early to evaluate the replicability of the ULLs in the UNaLab project, our data about the three cities (also drawing on previous experiences with ULLs) suggests that clear opportunities for replicating the findings are needed to motivate adoption of the ULL approach. The potential for upscaling specific NBS innovations partially depends upon the ability to capture and learn from their outcomes. Barriers related to upscaling and/or replication can also pertain to the local organisational culture and the prevailing perceptions of the urban authorities involved [37]. Successful replication of ULLs needs a strong community of stakeholders from various sectors, departments and institutions, which facilitates the dissemination of key learnings and experiences.

- $\quad$ Lack of learning from other experiences

Inadequate opportunities to share the lessons learned and experiences in similar projects is another barrier limiting the adoption of ULLs for NBS. A number of knowledge repositories such as Oppla (https: / / oppla.eu/, accessed on 1 October 2021) and the Urban Nature Atlas (https://una.city/, accessed on 1 October 2021) were recently developed to collect information about experiences of different cities across the world with NBS co-creation. However, our workshop and interview data imply these platforms are not yet widely known and used by urban stakeholders. In addition, several interviewees pointed at the need for more interactive opportunities for knowledge exchange across different cities.

\subsubsection{Ethical Barriers}

- Intellectual property (IP)

One of the ethical barriers is the engagement of the private sector in the co-creation process [38]. Participants from the private companies contributing to the ULLs were frequently not willing to share specific ideas on possible solutions and actions. Companies were often reluctant to share their intellectual property (IP). Moreover, there was no explicit protocol regarding IP in the ULLs, which tends to inhibit the co-creation process.

- $\quad$ Privacy issues

The protection of citizens' privacy has been a critical challenge for the cities involved in the UNaLab project [27]. For example, the municipality of Eindhoven had a plan to observe and monitor the movements and activities of citizens in and around the Clausplein (i.e., the site of a newly developed NBS), using Lidar cameras, to assess the social functionality of the NBS implemented. However, the cameras could not be installed because they did not comply with the privacy regulations in place in the Netherlands. 


\section{- Inclusiveness}

Finally, inclusiveness was identified as a key ethical issue, as not all relevant stakeholders were represented in the ULL co-creation processes in the three cities [27]. The typical participant was an individual that would frequently attend municipal meetings, and the desired diversity of participants with respect to ethnicity, age and level of income was not accomplished.

\section{Analysis and Modelling of Barriers}

The barriers identified in the previous section are highly interconnected, and understanding these interdependencies is necessary for enabling cities to develop effective roadmaps. For this purpose, Interpretive Structural Modelling (ISM) is used to identify the relationships among the barriers and develop a structural model. This method was used previously to analyse the barriers for the implementation of NBS [13]. In the following subsections, the results of a survey regarding the impact of barriers are presented, the various steps taken with regard to ISM are outlined, and the final results are presented.

In order to analyse the barriers more deeply, 16 barriers were selected from the list in Section 3.2 for further analysis (see Table 1). These barriers were identified in the collective workshop as the most frequent problems limiting the adoption of ULLs. For example, various (e.g., privacy-related) ethical barriers were eliminated from the list because they were only experienced in a single project in one of three cities, whereas all other barriers selected were present in at least two cities. We also needed to somewhat reduce the list generated in Section 3.2 because the ISM method can only handle a moderate number of variables (see Section 4.2).

Table 1. Barriers for ULL adoption for NBS: survey results $(N=25)$.

\begin{tabular}{|c|c|c|c|}
\hline \# & Barriers & Average Rate (from 1 to 10 ) & Rank \\
\hline B1 & Lack of sustainable financial resources & 7.6 & 1 \\
\hline B2 & Uncertainties regarding the added value and benefits of ULLs & 7.3 & 2 \\
\hline B3 & Lack of political will and long-term commitment & 7.2 & 3 \\
\hline B4 & Disconnection from the mainstream development process & 7.1 & 4 \\
\hline B5 & NBS monitoring and assessment challenges & 6.9 & 5 \\
\hline B6 & Sectoral silos & 6.6 & 6 \\
\hline B7 & Lack of engagement to take responsibility & 6.4 & 7 \\
\hline B8 & Inflexible hierarchical organisational structure & 6.1 & 8 \\
\hline B9 & Lack of public awareness and engagement & 6 & 9 \\
\hline $\mathrm{B} 10$ & Lack of skilled knowledge brokers & 5.9 & 10 \\
\hline B11 & Risk aversion and reluctance to change & 5.9 & 10 \\
\hline $\mathrm{B} 12$ & Lack of supportive legal and policy frameworks & 5.8 & 12 \\
\hline $\mathrm{B} 13$ & Lack of available guidelines and tools for engagement & 5.8 & 12 \\
\hline B14 & Conflicting expectations among urban stakeholders & 5.6 & 14 \\
\hline B15 & Lack of learning from other experiences & 5.6 & 14 \\
\hline B16 & Perceived complexity of the ULL approach & 3.8 & 16 \\
\hline
\end{tabular}

\subsection{Survey}

In an online workshop on 24 November 2020, including all partners involved in the UNaLab project, a survey was used to determine how the participants rate the importance of the 16 main barriers and how these barriers influenced their living labs. The result of the survey is presented in Table 1 . In this table, barriers are ranked based on the average rating by participants.

Except for "perceived complexity of the ULL approach", the average rating given by the expert group was greater than 5 for all barriers, which underpins the relevance of the barriers selected. The most critical barrier appears to be the lack of financial resources sustained over a long time period, followed by uncertainties regarding the added values and benefits provided by ULLs and the lack of political commitment. In the next subsection, we will analyse how these barriers affect one another. 


\subsection{Interpretive Structural Modelling}

Interpretive Structural Modelling (ISM) is a method that serves to identify the interdependencies among different elements of a complex system and provide a systemic model by decomposing this system into several segments [39]. ISM draws on the knowledge and experience of experts to develop a collective understanding of the interdependencies. It primarily operates as a group learning process, which fits the collaborative and co-creative nature of this study [40].

ISM has some limitations. Only a limited number of variables can be considered, as a large number of variables can over-complicate the model and make it difficult to understand the outcomes [41]. Therefore, some of the identified barriers were eliminated from the list, as described earlier. The ISM method includes eight steps:

1. Identifying the variables affecting the system studied;

2. Establishing a relationship between each pair of variables identified in step 1;

3. Developing the Structural Self-Interaction Matrix (SSIM);

4. Developing the reachability matrix from the SSIM and checking this matrix for transitivity;

5. Partitioning the reachability matrix obtained in step 4 into different levels;

6. Drawing a directed graph (or digraph) based on the identified relationships and removing the transitivity links;

7. Converting the digraph into an ISM model by replacing nodes of the variables with statements;

8. Using MICMAC analysis to classify barriers based on their driving and dependence power.

Step 1, which involves identifying the barriers, has already been reported. Step 2 involves identifying the relationships between the barriers. These relationships were identified in the collective workshop conducted on 28 November 2019. Experts were asked to discuss and identify the interdependencies among the barriers. These participants were also invited to define the direction of cause-effect relationships among various barriers. The identified relationships are presented in Table S1 in the Supplementary Materials. Steps 3 to 6 are also discussed in greater detail in the Supplementary Materials. The results of steps 7 and 8 are presented in the remainder of this section.

\subsubsection{ISM-Based Model}

After analysing the interdependencies among identified barriers, the ISM model was generated. This model has six levels, shown in Figure 1. Level I includes the lack of engagement to take responsibility (B7). The lack of political will and long-term commitment (B3) is located on Level VI, at the bottom of the model. A detailed explanation of the process of classifying the barriers into different levels can be found in the Supplementary Materials. The arrows reflect the direction of the relationships among barriers. The arrow between barriers $i$ and $j$ means that $j$ is affected by $i$. Each barrier, at a higher level, is directly affected by at least one barrier at the next lower level and indirectly affected by many other lower-level barriers. For example, B3 is directly or indirectly affecting all other barriers, while B7 is affected by all other barriers in the model; another example is the lack of skilled knowledge brokers (B10) and sectoral silos (B6), which are directly or indirectly affected by B3, B4, B8 and B12 and (in)directly affect all other barriers. 


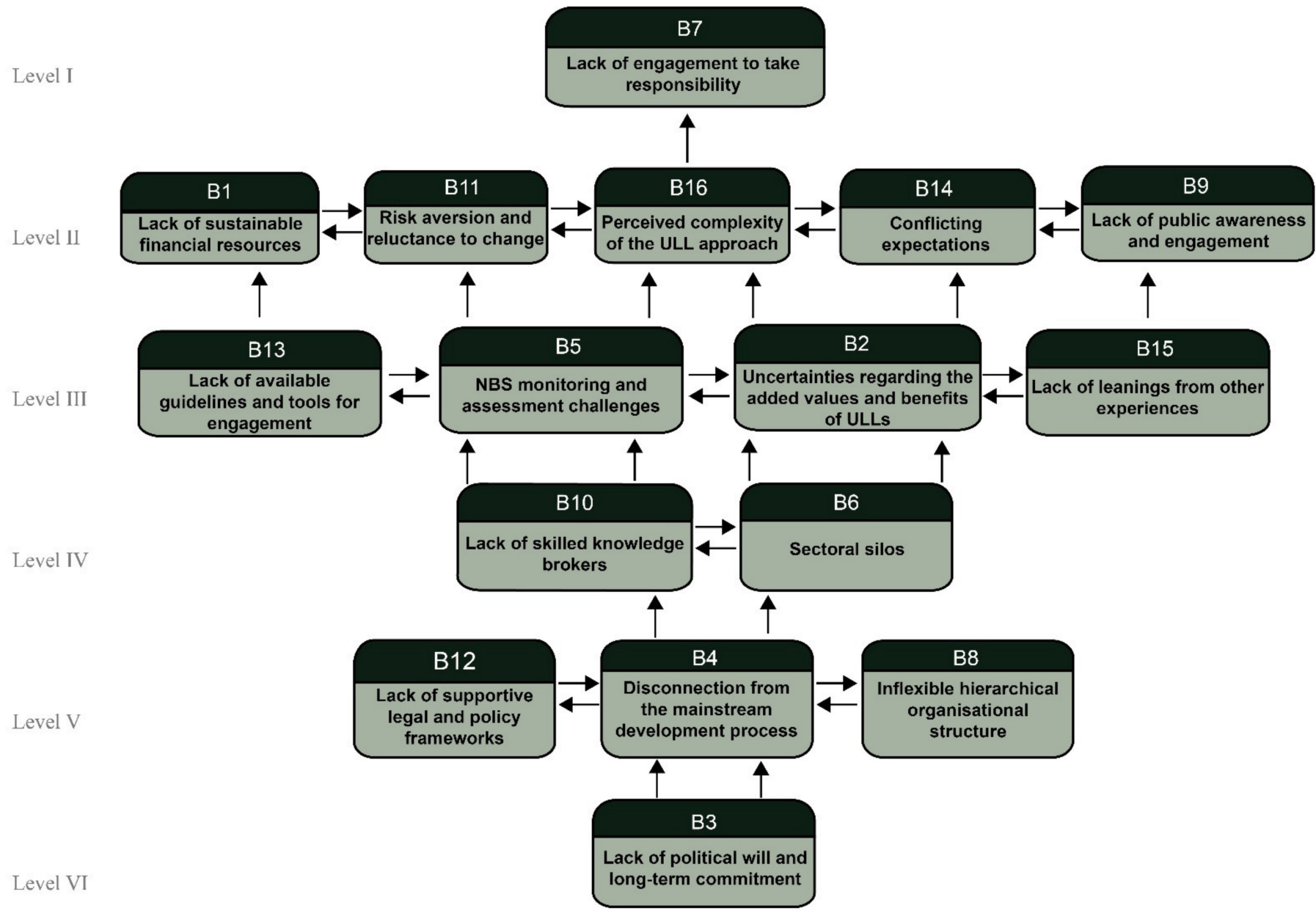

Figure 1. The barriers model based on ISM.

\subsubsection{MICMAC Analysis}

Cross-impact matrix multiplication applied to classification (Matrice d'Impacts CroisesMultiplication Appliqúe an Classment) is also known as MICMAC analysis [41]. MICMAC analysis serves to differentiate barriers considering their driving and dependence powers, which were identified in the final reachability matrix (see Supplementary Materials). The driving power of each barrier is the total number of barriers (including itself) which it may affect, while dependence power is the total number of barriers which may affect it. Accordingly, the 16 barriers are classified among four categories in Figure 2:

1. Quadrant I: Autonomous barriers are barriers with both weak dependence and driving power. This type of barrier is rather disconnected from the rest. None of the barriers in this study appear to be in this category.

2. Quadrant II: Dependent barriers have strong dependence power but low driving power.

3. Quadrant III: Linkage barriers have strong driving power and strong dependence power. Linkage barriers tend to be unstable, meaning any action on any of them is likely to affect several other barriers and cause a feedback mechanism.

4. Quadrant IV: Driving (or independent) barriers have strong driving power, but weak dependence power. 


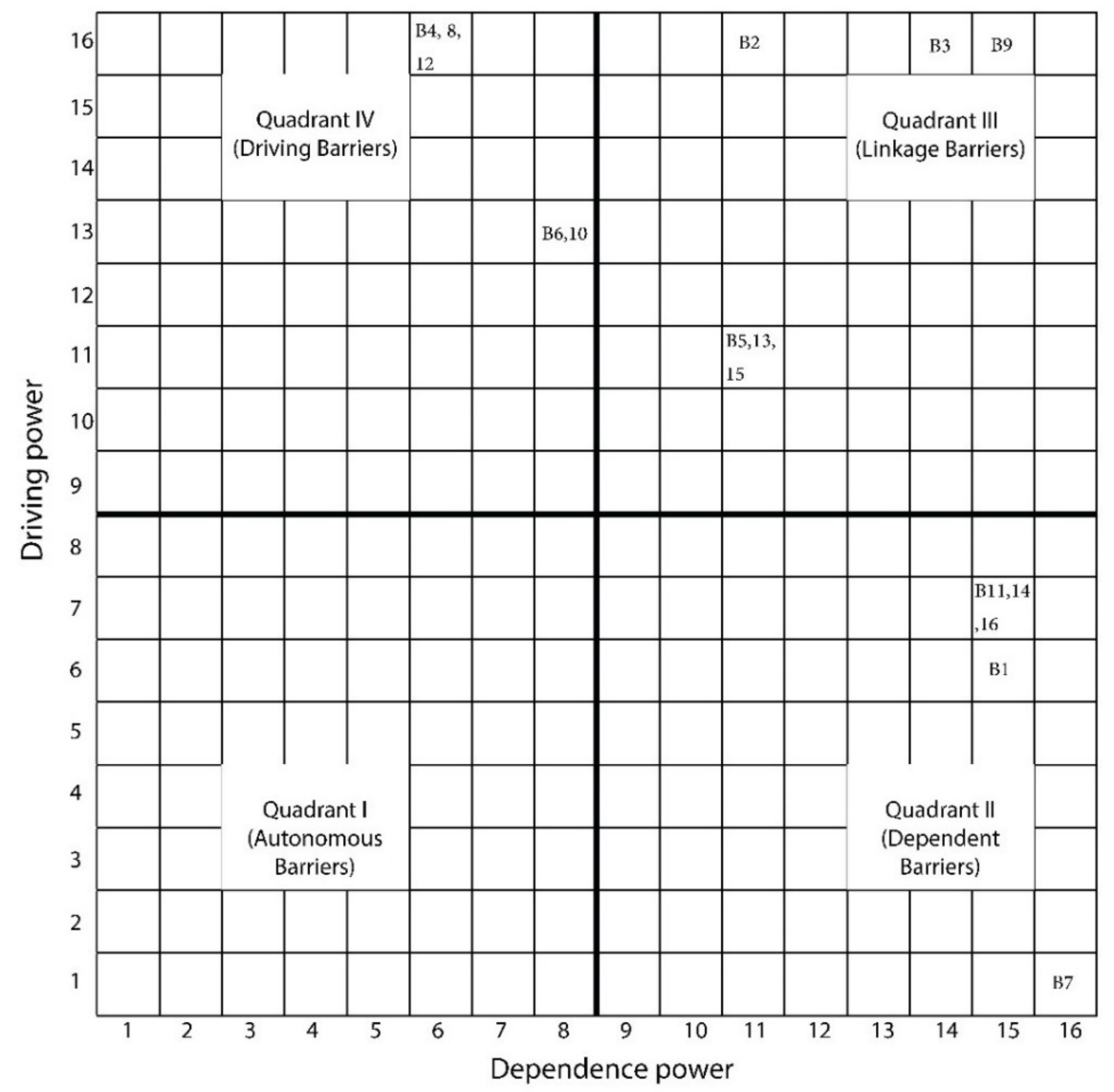

Figure 2. MICMAC analysis of the barriers.

Generally, the barriers located in Quadrant IV in Figure 2 are at the bottom of the ISM model in Figure 1, whereas barriers located in Quadrant III are at the middle parts of the model in Figure 1, and barriers located in Quadrant II are placed at the upper parts of the ISM model. However, there are a few exceptions to this general observation. The results are discussed in more detail in the next section.

\section{Discussion}

\subsection{Analysis of the Results}

There are five barriers located in Quadrant IV of Figure 2:

- Disconnection from the mainstream development process (B4);

- Sectoral silos (B6);

- Inflexible hierarchical organisational structure (B8);

- $\quad$ Lack of skilled knowledge brokers (B10);

- Lack of supportive legal and policy frameworks (B12).

The barriers B4, B8 and B12 are relatively independent but do have a significant impact on other barriers. This high impact can also be inferred from the ISM model in Figure 1. These three barriers mainly arise from the institutional characteristics of municipalities. Moreover, the presence of B6 and B10 in this quadrant also points at the pivotal role of developing and sustaining good connections among different departments and/or brokers that make such connections. In Figure 1, B6 and B10 directly affect the knowledge-related barriers at level III. Effective collaboration among stakeholders across disciplines and scales is therefore instrumental in growing the level of knowledge of stakeholders as well as developing integral co-creation and monitoring plans.

Six barriers are located in Quadrant III of Figure 2:

- Uncertainties regarding the added values and benefits of ULLs (B2); 
- $\quad$ Lack of political will and long-term commitment (B3);

- $\quad$ NBS monitoring and assessment challenges (B5);

- Lack of public awareness and engagement (B9);

- Lack of available guidelines and tools for engagement (B13);

- Lack of learning from other experiences (B15).

B3 is located at the bottom of the ISM model, while it is in Quadrant III of Figure 2. This implies that B3 is a significant barrier, affected by other barriers, especially those also in Quadrant III. As observed earlier, the barriers in this quadrant play a central role. It is therefore very important to pay attention to the feedback loops among the barriers when making plans to address them. Moreover, the presence of the knowledge-related barriers B2, B5, B13 and B15 in this quadrant suggests that improving the awareness of urban stakeholders and providing opportunities to enhance the stakeholders' knowledge about ULLs for NBS is essential to address and overcome all other barriers.

Specific attention should be paid to the lack of public awareness and engagement (B9). Citizens play a central role in ULLs, and our analysis shows that the lack of public awareness and engagement significantly undermines the adoption of ULLs. Improving public awareness and engagement can thus help to improve the city's political and institutional environment and enhance the motivation of citizens and other urban stakeholders to support ULLs and commit to them.

Finally, five barriers are located in the Quadrant II of Figure 2:

- Lack of sustainable financial support (B1);

- Lack of engagement to take responsibility (B7);

- Risk aversion and reluctance to change (B11);

- Conflicting expectations (B14);

- Perceived complexity of the ULL approach (B16).

These barriers, which are mostly cognitive and behavioural in nature, are located at levels I and II in Figure 1. To address these barriers strategically, one needs to consider the other barriers affecting them. One notable point here is the presence of B1 as an organisational and structural barrier in this quadrant. B1 was identified as the most important barrier in Table 1 and is highly affected by several other barriers. However, it does not imply that the lack of financial support is a less important barrier, but rather that effectively addressing this barrier requires one to overcome several other barriers which are affecting it [42].

Whereas a previous study [13] served to explore and model barriers to NBS implementation, this study analyses the barriers to adopting ULLs for NBS. There are several similarities between the results of the two studies, mainly because both the ULL and NBS concepts are innovative approaches that deal with the social, ecological and technological aspects of urban life. For example, both studies find the political and institutional barriers to be driving other barriers, and knowledge barriers are found to be pivotal linkages, that is, addressing them can generate positive feedback loops which help address various other barriers. Moreover, both studies conclude that cognitive and behavioural barriers are dependent in nature.

However, there are also key differences between the results of this study and the previous one [13]. The most important difference concerns the key role of effective collaboration across policy domains and scales as well as the role that knowledge brokers and intermediaries can play in adopting and developing ULLs. We will further discuss these findings in the next subsection.

\subsection{Addressing the Barriers}

Adopting the ULL approach can provide a suitable institutional environment to successfully develop and realise the multifunctionality of NBS. However, several organisational, structural, knowledge, cognitive and ethical barriers tend to undermine the ability of cities to adopt ULLs for NBS implementation. Using the ISM method, we provide a systemic understanding in this article to help cities develop more effective strategic plans 
for addressing the various barriers. The resulting model shows that the barriers are not isolated; addressing one of them can have a major impact on several other barriers. However, addressing multiple barriers requires more effort and time and calls for more involvement of actors from different institutional levels.

For example, addressing the political and institutional barriers in cities is critical $[43,44]$ but also requires many more resources to overcome them. These barriers are mostly a legacy from the past, which makes them rather difficult to tackle for municipal staff and local politicians. To overcome this type of barrier, legislative and other political changes at a higher institutional level are necessary $[45,46]$.

A critical point in the model is where knowledge and organisational barriers meet. Here, sectoral silos and the lack of knowledge brokers are two central barriers. The pivotal role of knowledge brokers and other intermediary actors in sustainability transitions has been widely studied and acknowledged [47-49]. As shown in the ISM model presented in the previous section, knowledge brokers play an important role in generating effective networks, advocating innovations and translating the (knowledge about) NBS across scales and disciplines [35]. Intermediaries can also play an essential role in connecting financiers with other actors and stakeholders. They can facilitate collaborations by fostering the knowledge flow to reduce uncertainties [42]. Providing favourable conditions for intermediary actors and knowledge brokers should be prioritised in municipalities seeking to adopt ULLs for NBS implementation. However, as shown in our model, a supportive institutional environment is necessary for enabling intermediaries to engage and progress [35]. In the ULLs in the three cities studied in this paper, several intermediary actors were present; in terms of the typology proposed by Kivimaa et al. [49], these actors can be primarily categorised as regime-based intermediaries that are bounded to existing institutional arrangements but are inclined or mandated to build partnerships and promote transition. In the course of the UNaLab project, these intermediaries played a central role in providing opportunities for collaboration and collective exploration of ideas.

Other actions addressing the knowledge-related barriers can also be important. In order to facilitate knowledge exchange, several tools and approaches were introduced in the UNaLab project, including an online platform (with a co-creation toolkit) which was designed to share knowledge and support stakeholders in finding suitable approaches for living lab co-creation of NBS. As such, future work in this area will need to evaluate the effectiveness of such tools. Most importantly, our findings suggest that any improvements in knowledge and awareness can spark a virtuous feedback cycle that helps to address many other barriers.

\section{Conclusions}

ULLs appear to provide a safe and supportive environment for collaboration and experimentation with NBS. However, the adoption of ULLs often suffers from several barriers. In this paper, we identified the main barriers in this area and explored the interdependencies among them using ISM modelling and MICMAC analysis. We generated a hierarchical model that shows the interdependencies among the barriers. Our findings suggest that a systemic understanding of these barriers is critical for a supportive political and institutional setting for the adoption of ULLs. Moreover, knowledge brokers and intermediaries appear to play a pivotal role also in reducing the influence of sectoral silos on the adoption of ULLs. Notably, the general model of barriers arising from this study enables the development of city-specific models that are more context-sensitive in terms of the local institutional, political and other conditions.

Supplementary Materials: The following are available online at https:/ / www.mdpi.com/article / 10.3390/su132313276/s1, Table S1: Structural self-interaction matrix (SSIM); Table S2: Initial Reachability Matrix; Table S3: Final reachability matrix; Table S4: First iteration; Table S5: Second iteration; Table S6: Third iteration; Table S7: Fourth iteration; Table S8: Fifth iteration; Table S9: Sixth iteration. 
Author Contributions: Conceptualization, S.S.; methodology, S.S.; formal analysis, S.S.; writing—original draft preparation, S.S.; writing-review and editing, S.S., Q.H., A.G.L.R., B.d.V., R.V., E.d.O., S.Z. and L.W. All authors have read and agreed to the published version of the manuscript.

Funding: This research has received funding from the European Union's Horizon 2020 Research and Innovation Programme under Grant Agreement No. 730052.

Institutional Review Board Statement: Ethical review and approval were waived for this study, because Eindhoven University of Technology only established its Ethical Review Board after this study was initiated and approved by the Review Board of the department of the Built Environment of this university (d.d. 26 February 2019).

Informed Consent Statement: Informed consent was obtained from all subjects involved in the study.

Acknowledgments: We would like to thank all UNaLab partners for their participation in several workshops and sharing their insights.

Conflicts of Interest: The authors declare no conflict of interest.

\section{References}

1. Kabisch, N.; Stadler, J.; Korn, H.; Bonn, A.; Frantzeskaki, N.; Pauleit, S.; Naumann, S.; Davis, M.; Artmann, M.; Haase, D.; et al. Nature-Based Solutions to Climate Change Mitigation and Adaptation in Urban Areas: Perspectives on Indicators, Knowledge Gaps, Barriers, and Opportunities for Action. Ecol. Soc. 2016, 21, 39. [CrossRef]

2. Lafortezza, R.; Chen, J.; van den Bosch, C.K.; Randrup, T.B. Nature-Based Solutions for Resilient Landscapes and Cities. Environ. Res. 2018, 165, 431-441. [CrossRef] [PubMed]

3. European Commission Towards an EU Research and Innovation Policy Agenda for Nature-Based Solutions \& Re-Naturing Cities: Final Report of the Horizon 2020 Expert Group on Nature-Based Solutions and Re-Naturing Cities; European Commission: Brussels, Belgium, 2015.

4. Cohen-Shacham, E.; Walters, G.; Janzen, C.; Maginnis, S. Nature-Based Solutions to Address Global Societal Challenges; IUCN: Gland, Switzerland, 2016.

5. Dumitru, A.; Wendling, L. Evaluating the Impact of Nature-Based Solutions: A Handbook for Practitioners; European Commission: Brussels, Belgium, 2021.

6. $\quad$ Raymond, C.M.; Berry, P.; Breil, M.; Nita, M.R.; Kabisch, N.; de Bel, M.; Enzi, V.; Frantzeskaki, N. An Impact Evaluation Framezork to Support Planning and Evaluation of Nature-Based Solutions Projects; EKLIPSE: Leipzig, Germany, 2017.

7. Collier, M.; Connop, S. Urban Living Labs: Nature-Based Solutions Experiences in the EU. In Soluções Baseadas na Natureza e os Desafios da Água: Accelerando a Transição Para Cidades Mais Sustentáveis; European Commission-Directorate-General for Research and Innovation: Brussels, Belgium, 2020; ISBN 978-92-76-18445-4.

8. Ascione, G.S.; Cuomo, F.; Mariotti, N.; Corazza, L. Urban Living Labs, Circular Economy and Nature-Based Solutions: Ideation and Testing of A New Soilin the City of Turin Using a Multi-Stakeholder Perspective. Circ. Econ. Sustain. 2021, 1, 545-562. [CrossRef]

9. Von Wirth, T.; Fuenfschilling, L.; Frantzeskaki, N.; Coenen, L. Impacts of Urban Living Labs on Sustainability Transitions: Mechanisms and Strategies for Systemic Change through Experimentation. Eur. Plan. Stud. 2019, 27, 229-257. [CrossRef]

10. Ståhlbröst, A.; Habibipour, A.; Chronéer, D.; Vaittinen, I.; Zalokar, S.; Mafe, C. UnaLab ULL Framework. D2.1; European Commission: Brussels, Belgium, 2018.

11. Nevens, F.; Frantzeskaki, N.; Gorissen, L.; Loorbach, D. Urban Transition Labs: Co-Creating Transformative Action for Sustainable Cities. J. Clean. Prod. 2013, 50, 111-122. [CrossRef]

12. Lupp, G.; Zingraff-Hamed, A.; Huang, J.J.; Oen, A.; Pauleit, S. Living Labs-A Concept for Co-Designing Nature-Based Solutions. Sustainability 2021, 13, 188. [CrossRef]

13. Sarabi, S.; Han, Q.; Romme, A.G.L.; de Vries, B.; Valkenburg, R.; den Ouden, E. Uptake and Implementation of Nature-Based Solutions: An Analysis of Barriers Using Interpretive Structural Modeling. J. Environ. Manag. 2020, 270, 110749. [CrossRef]

14. Särkilahti, M.; Mustajärvi, K.; Leppänen, S. Biosuodattimia, Hevoshakoja Ja Viherkattoja-Yhdessä Oppiminen Luontopohjaisten Ratkaisujen Luomisessa. Alue Ympäristö 2019, 48, 20-37. [CrossRef]

15. Hawxwell, T.; Mok, S.; Maciulyte, E.; Sautter, J.; Effenberger, N.; Dobrokhotova, E.; Suska, P. Municipal Governance Recommendations; European Commission: Brussels, Belgium, 2019.

16. van Dinter, M.; Habibipour, A. D2.2-Co-Creation Workshops Report; European Commission: Brussels, Belgium, 2019.

17. Dhakal, K.P.; Chevalier, L.R. Managing Urban Stormwater for Urban Sustainability: Barriers and Policy Solutions for Green Infrastructure Application. J. Environ. Manag. 2017, 203, 171-181. [CrossRef]

18. Eisenack, K.; Moser, S.C.; Hoffmann, E.; Klein, R.J.T.; Oberlack, C.; Pechan, A.; Rotter, M.; Termeer, C.J.A.M. Explaining and Overcoming Barriers to Climate Change Adaptation. Nat. Clim. Change 2014, 4, 867. [CrossRef]

19. Johns, C.M. Understanding Barriers to Green Infrastructure Policy and Stormwater Management in the City of Toronto: A Shift from Grey to Green or Policy Layering and Conversion? J. Environ. Plan. Manag. 2019, 62, 1377-1401. [CrossRef] 
20. Hawxwell, T.; Mok, S.; Mačiulytė, E.; Sautter, J.; Dobrokhotova, E. Municipal Governance for Nature-Based Solutions; Fraunhofer IAO: Stuttgart, Germany, 2019.

21. URBAN GreenUP D1.5-Barriers and Boundaries Identification; European Commission: Brussels, Belgium, 2018.

22. Nesshöver, C.; Assmuth, T.; Irvine, K.N.; Rusch, G.M.; Waylen, K.A.; Delbaere, B.; Haase, D.; Jones-Walters, L.; Keune, H.; Kovacs, E.; et al. The Science, Policy and Practice of Nature-Based Solutions: An Interdisciplinary Perspective. Sci. Total Environ. 2017, 579, 1215-1227. [CrossRef]

23. Gascó, M. Living Labs: Implementing Open Innovation in the Public Sector. Gov. Inf. Q. 2017, 34, 90-98. [CrossRef]

24. Connecting Nature Financing and Business Models. Available online: https:/ / connectingnature.eu/financing-and-businessmodels (accessed on 1 August 2021).

25. Søndergaard, H.; Burcharth, A. Open Innovation Practices and Implementation Barriers: Unwillingness to Receive and Share Knowledge. In Proceedings of the DRUID Society Conference, Copenhagen, Denmark, 15-17 June 2011; p. 8.

26. Chalmers, D. Social Innovation: An Exploration of the Barriers Faced by Innovating Organizations in the Social Economy. Local Econ. 2012, 28, 17-34. [CrossRef]

27. Van den Brink, M.; Fidder, R.; Remmers, J.; Schoonderbeek, J. An Urban Living Lab Approach for the Implementation of Climate Adaptation Measures; Wageningen University and Research: Wageningen, The Netherlands, 2017.

28. Sarabi, S.; Han, Q.; Romme, A.G.L.; de Vries, B.; Wendling, L. Key Enablers of and Barriers to the Uptake and Implementation of Nature-Based Solutions in Urban Settings: A Review. Resources 2019, 8, 121. [CrossRef]

29. Wamsler, C.; Wickenberg, B.; Hanson, H.; Alkan Olsson, J.; Stålhammar, S.; Björn, H.; Falck, H.; Gerell, D.; Oskarsson, T.; Simonsson, E.; et al. Environmental and Climate Policy Integration: Targeted Strategies for Overcoming Barriers to Nature-Based Solutions and Climate Change Adaptation. J. Clean. Prod. 2020, 247, 119154. [CrossRef]

30. BearingPoint Accelerating Open Innovation in the Public Sector; BearingPoint: Amsterdam, The Netherlands, 2016.

31. Mok, S.; Mačiulytè, E.; Bult, P.H.; Hawxwell, T. Valuing the Invaluable(?)—A Framework to Facilitate Stakeholder Engagement in the Planning of Nature-Based Solutions. Sustainability 2021, 13, 2657. [CrossRef]

32. Wendling, L.; Rinta-Hiiro, V.; Jermakka, J.; Fatima, Z.; Malin, z.-C.R.; Ascenso, A.; Miranda, A.I.; Roebeling, P.; Martins, R.; Mendonça, R. NBS Performance and Impact Monitoring Protocols; European Commission: Brussels, Belgium, 2019.

33. Dubovik, M.; Rinta-Hiiro, V.; zu-Castell Rüdenhausen, M.; Wendling, L.; Laikari, A.; Jakstis, K.; Fischer, L.K.; Spinnato, P.; Jermakka, J.; Fatima, Z.; et al. Preliminary Nature-Based Solutions Implementation Handbook; European Commission: Brussels, Belgium, 2020.

34. Dumitru, A.; Wendling, L. Evaluating the Impact of Nature-Based Solutions Appendix of Methods; European Commission: Brussels, Belgium, 2021.

35. Frantzeskaki, N.; Bush, J. Governance of Nature-Based Solutions through Intermediaries for Urban Transitions-A Case Study from Melbourne, Australia. Urban For. Urban Green. 2021, 64, 127262. [CrossRef]

36. Hakkarainen, L.; Hyysalo, S. The Evolution of Intermediary Activities: Broadening the Concept of Facilitation in Living Labs. Technol. Innov. Manag. Rev. 2016, 6, 45-58. [CrossRef]

37. Junginger, S. Design Legacies: Why Service Designers Are Not Able to Embed Design in the Organization. In Proceedings of the 4th Conference on Service Design and Service Innovation, Lancaster, UK, 9-11 April 2014; Lancaster University: Lancaster, UK, 2014; pp. 164-172.

38. Mergel, I. Open Innovation in the Public Sector: Drivers and Barriers for the Adoption of Challenge.Gov. Public Manag. Rev. 2018, 20, 726-745. [CrossRef]

39. Sindhu, S.; Nehra, V.; Luthra, S. Identification and Analysis of Barriers in Implementation of Solar Energy in Indian Rural Sector Using Integrated ISM and Fuzzy MICMAC Approach. Renew. Sustain. Energy Rev. 2016, 62, 70-88. [CrossRef]

40. Raj, T.; Shankar, R.; Suhaib, M. An ISM Approach for Modelling the Enablers of Flexible Manufacturing System: The Case for India. Int. J. Prod. Res. 2008, 46, 6883-6912. [CrossRef]

41. Attri, R.; Dev, N.; Sharma, V. Interpretive Structural Modelling (ISM) Approach: An Overview. Res. J. Manag. Sci. 2013,2 , 3-8.

42. Polzin, F.; von Flotow, P.; Klerkx, L. Addressing Barriers to Eco-Innovation: Exploring the Finance Mobilisation Functions of Institutional Innovation Intermediaries. Technol. Forecast. Soc. Change 2016, 103, 34-46. [CrossRef]

43. Kinzer, K. How Can We Help? An Exploration of the Public's Role in Overcoming Barriers to Urban Sustainability Plan Implementation. Sustain. Cities Soc. 2018, 39, 719-728. [CrossRef]

44. Qiao, X.-J.; Liu, L.; Kristoffersson, A.; Randrup, T.B. Governance Factors of Sustainable Stormwater Management: A Study of Case Cities in China and Sweden. J. Environ. Manag. 2019, 248, 109249. [CrossRef] [PubMed]

45. Moser, S.C.; Ekstrom, J.A. A Framework to Diagnose Barriers to Climate Change Adaptation. Proc. Natl. Acad. Sci. USA 2010, 107, 22026-22031. [CrossRef]

46. Ekstrom, J.A.; Moser, S.C. Identifying and Overcoming Barriers in Urban Climate Adaptation: Case Study Findings from the San Francisco Bay Area, California, USA. Urban Clim. 2014, 9, 54-74. [CrossRef]

47. Agogué, M.; Yström, A.; le Masson, P. Rethinking the Role of Intermediaries as an Architect of Collective Exploration and Creation of Knowledge in Open Innovation. Int. J. Innov. Manag. 2013, 17, 1350007. [CrossRef]

48. Kanda, W.; Kuisma, M.; Kivimaa, P.; Hjelm, O. Conceptualising the Systemic Activities of Intermediaries in Sustainability Transitions. Environ. Innov. Soc. Transit. 2020, 36, 449-465. [CrossRef]

49. Kivimaa, P.; Boon, W.; Hyysalo, S.; Klerkx, L. Towards a Typology of Intermediaries in Sustainability Transitions: A Systematic Review and a Research Agenda. Res. Policy 2019, 48, 1062-1075. [CrossRef] 